\title{
Women's Empowerment and Infant and Young Child Feeding Practice in Low- and Middle-Income Countries: A Systematic Review
}

\author{
Eka Mishbahatul Mar'ah $\operatorname{Has}^{1}$ Nursalam Nursalam ${ }^{2, *}$ Yuni Sufyanti Arief ${ }^{3}$
}

\author{
${ }^{1}$ Doctoral study program students, Faculty of Nursing (Universitas Airlangga), Surabaya, Indonesia \\ ${ }^{2}$ Lecturer, Department of Basic, Medical-Surgical, and Critical Nursing, Faculty of Nursing (Universitas Airlangga), Surabaya, \\ Indonesia \\ ${ }^{3}$ Lecturer, Department of Maternity and Paediatric Nursing, Faculty of Nursing (Universitas Airlangga), Surabaya, Indonesia \\ *Corresponding author. Email: nursalam@fkp.unair.ac.id
}

\begin{abstract}
Adherence to the WHO guidelines for infant and young child feeding (IYCF) practice in low- and middle-income countries (LMIC) is unsatisfactory. Women's empowerment has been noticed as the key factor which mediating intra-household resources to achieve optimal IYCF practice. Thus, we undertook a systematic review of studies exploring the indicator of women's empowerment, which influenced IYCF practice. Keyword searches were conducted in Scopus, CINAHL, Science Direct, PubMed, and Google Scholar using combinations of the following terms: empowerment, women, feeding behaviour, and infant. The bibliography was also searched for potential publications. Studies were included if involved children $<2$ years old and their mother/carer as participants, conducted in LMIC, published from 2011 or later, and English. Researchers performed study selection, data extraction, quality appraisal, and thematic analysis. From 17861 results, twenty-four studies were included. Mostly, socioeconomic status and education used as the indicator of women's empowerment in IYCF practice. We found women's empowerment indicators that facilitate IYCF practices, namely a sense of leadership, high education, land ownership, involvement in household decision-making processes, against domestic violence, high socioeconomic status, and employment. Meanwhile, the indicators that inhibit uneducated or low education, forced to be head of the family, not involved in the decisionmaking process in family, low socioeconomic status, and high workload. Women's autonomy over resources is debatable as a facilitator or barrier, as we found inconsistent results. This review highlighted the indicator of women empowerment, which is important for proper IYCF practice. Future research and empowerment intervention to improve IYCF should address these findings.
\end{abstract}

Keywords: women's empowerment, infant and young child feeding practice, low- and middle-income countries

\section{INTRODUCTION}

Childhood malnutrition continues to be a major public health problem in the world [1]. By 2020, 144 million children under five years old are stunted, 47 million are wasted, and 38 million are overweight [2]. The burden of childhood malnutrition is greatest in LMIC, especially in Africa and Asia. While the prevalence of childhood stunting and wasting is slowly declining, the prevalence of childhood overweight and obesity increases [3]. This progress is inadequate to reach the target of the World Health Assembly by 2025 and the Sustainable Development Goals by 2030 [4].

Directly, childhood malnutrition was caused by inadequate dietary intakes and diseases [5]. A child's improper dietary intakes and frequent exposure to the diseases are affected by several underlying factors, including household insecure access to food, improper care and feeding practices, unhealthy household and environments, and inaccessible health care services [6], [7]. Besides that, the local politics and cultural environment also have a role in childhood malnutrition [8].

An unbalanced child's nutritional intake and needs can lead to malnutrition [9]. The critical period for childhood malnutrition is 6-23 months old when complementary food starts to be introduced until the child can consume family food [10]. Achieving appropriate complementary feeding for infants and young children is an essential milestone for ensuring their healthy growth and development [11]. Conversely, poor complementary feeding practices during the first two years of life can lead to physical growth failure, intellectual disability, and developmental delay, reduce productivity, reduce endurance, and increase morbidity and mortality [5], [12], [13].

Complementary feeding should meet WHO guidelines for IYCF practice, includes minimum dietary diversity, minimum meal frequency, and minimum acceptable diet [14]. 
In fact, in most LMIC, only a few children receive nutritionally adequate and safe complementary food. It is often given in poor nutritional quality, too early or too late, less in portions, or inadequately fed in a timely manner[15].

Children still depend on adults for food. In most families, women are responsible for child's feeding [16], [17]. Women's empowerment has recently been pointed out as an important key to facilitate intra-household resources owned by the family to be used optimally for proper IYCF practices [18]. Women empowerment refers to women's ability to decide and affect outcomes of importance to themselves and their families [19]. Kabeer divides women's empowerment dimension into resources, agency, and achievement [20]. Women who are empowered have these three things to claim the resources available in the household, have control, participate in decision-making, and act according to their wishes to change their lives to be better [21].

Previous research has shown a positive correlation between women's empowerment and children's nutritional status [21]-[25], as well as adequate IYCF practices with children's nutritional status [23]. However, not many studies have explored how the indicator of each women's empowerment dimensions influenced IYCF practices. This systematic review was conducted to explore the indicator of women's empowerment, which influenced IYCF practice. The scope of this review was focused on the evidence from LMIC.

\section{METHOD}

\subsection{Eligibility Criteria}

Studies that met the following criteria were included in this review.

Participants: children $<2$ years old and their mother/carer.

Outcomes: The WHO guidelines for IYCF practice (includes minimum dietary diversity, minimum meal frequency, and minimum acceptable diet).

Language: published in English.

Year: published from 2011 or later.

All study designs (quantitative/qualitative/mixed method) were included to ensure the diverseness of evidence. Studies focusing on exclusive breastfeeding only and review studies were excluded.

\subsection{Information Sources}

In July 2020, potential publications were searched on five major electronic databases, including Scopus, CINAHL, Science Direct, PubMed, and Google Scholar, according to the PRISMA (Preferred Reporting Items for Systematic Reviews and Meta-Analyses) statement [26]. A reference list of included articles was also hand-searched for potential publications. However, this review did not search grey literature to find additional potentials publications.

\subsection{Search Strategy}

The search strategy included the terms 'empowerment', 'women', 'feeding behaviour', 'determinant', and 'infant'. For example, the search strings used for Scopus were:

- Term 1: empowerment Empower* OR Empowerment

- Term 2: women Women OR Mother

- Term 3: feeding behavior

Feeding Behavior OR Food OR Complementary Feeding OR Feed OR Feeding Practice OR Nutrition

- Term 4: determinant

Determinant OR Associated Factor OR Factor

- Term 5: infant

Infant

\subsection{Study Selection and Data Extraction}

In total, 17861 titles and abstracts were screened according to inclusion criteria. Two researchers (EMMH and YS) assessed these articles independently for potential inclusion. Any discrepancies and disagreements were discussed and resolved through consensus or referral to the third researcher (NN) if the consensus could not be reached. The initial search identified 278 titles (Scopus [n=43], CINAHL [n=133], Science Direct [ $\mathrm{n}=22$ ], PubMed [ $\mathrm{n}=70]$, and Google Scholar $[\mathrm{n}=10])$ requiring review. After deleting 42 duplicated titles, 236 articles were processed for abstract review. As many as 182 articles were excluded cause of unrelated topics remaining 54 articles for full-text review. Only twenty-three articles were included in this review after the review of fulltext. The other 31 articles were excluded for reasons: 3 articles did not involve children $<2$ years old, 22 articles not focusing on IYCF practices, five articles conducted in highincome countries, and 1 article was missing. Reference list search of the 23 retained articles added one relevant article. Finally, 24 articles were included for data extraction (Figure 1). 


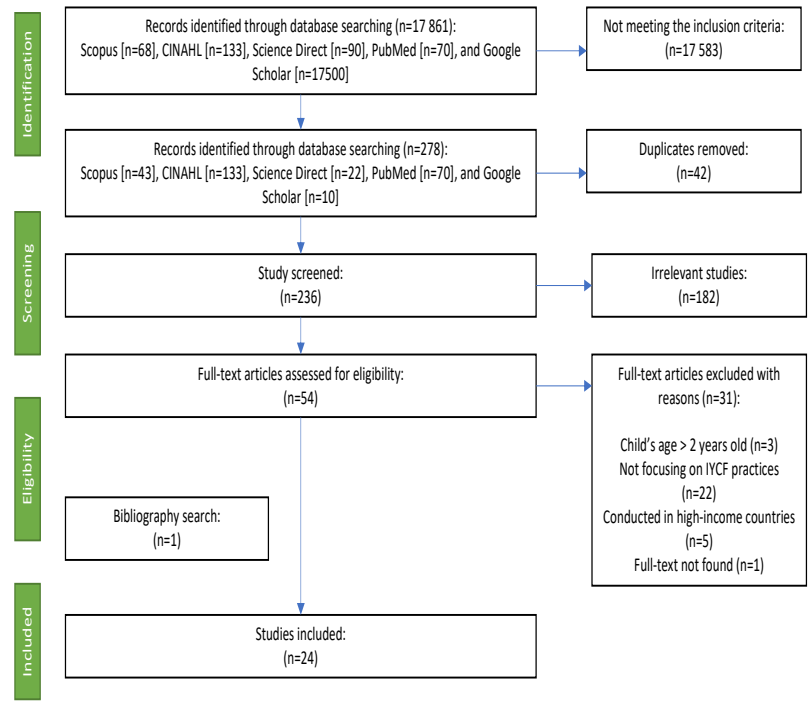

Figure 1 search flow diagram of the current systematic review

Data from the included studies were extracted in an Excel sheet to assist with data charting. Table 1 contains information about the title, authorship, study design, settings, number of participants, and key findings of the studies.

\subsection{The Assessment of Study Quality and Bias Risk}

To assess the methodological quality of each study, we use the JBI (Joanna Briggs Institute) Critical Appraisal Checklist, based on the study design. Three researchers perform critical appraisal independently. All of the disagreements during the assessment of study quality were discussed together to reach a consensus. Only studies that have critical appraisal scores of at least $50 \%$ were included in the review. As twenty-four studies get a score higher than $50 \%$, all of the studies were included in data synthesis. An assessment of bias risk of the found evidence did not critically investigate.

\subsection{Data Synthesis}

The findings of the included studies were then thematically analysed. The data synthesis results were described and explained following the study objectives.

\section{RESULT}

\subsection{Published Year}

The included articles were published in a varied year, but no such study was published in 2014. The majority, the included articles $(n=18 ; 75 \%)$ were published in the last five years.

\subsection{Origin}

The included articles were conducted in LMIC from six different geographical regions, include, West Africa, East Africa, Central Africa, South Asia, South-East Asia, and East Asia. Two studies were involved in various countries across South Asia and East Africa. Many of the studies were conducted in East Africa $(n=10 ; 41.67 \%)$.

\subsection{Number of Participants}

The total number of participants from 24 included studies was 80645 respondents, consist of 18161 children 0-2 years old; 40615 mothers; and 21678 infant-mother pairs.

\subsection{Study Design}

Out of the 24 included articles, two articles use qualitative study design [27], [28]. Among the quantitative study design articles, mostly was a cross-sectional study [13], [25], [29][47] and one study used quasi-experiment design [48]. A detailed explanation of the included articles can be seen in Table 1.

\subsection{Themes Emerged in The Review}

The current systematic review identified the indicator of women's empowerment, which can facilitate and inhibit IYCF practices.

\subsubsection{Women's empowerment as a facilitator of IYCF practices}

This review highlighted studies suggesting the indicator of women's empowerment which facilitate IYCF practices. These facilitators are as follows: 1) Resources, includes sense of leadership [39], well-educated [13], [30], [31], [37], [42], [43], [48], and land ownership [48]; 2) Agency, includes involvement in household decision making process [42], [48]; and 3) Achievement, includes women stands against domestic violence [41], high socioeconomic status [30], [31], [37], [38], [48], and employment [31].

This review identified the indicators of women's empowerment from the resources dimension, which related to the good practice of IYCF by mother. One study mentioned that maternal leadership, such as the sense of self and selfconfidence significantly associated with a greater dietary diversity score among female children [39]. Furthermore, this review identified seven studies that stated that maternal education was significantly correlated with the child's minimum dietary diversity and acceptable diet. At least, women should reach the finished secondary education level. As high as their level of education, their ability to perform a good IYCF practice is also increasing [13], [30], [31], [37], [42], [43], [48]. One study conducted in Ethiopia revealed that maternal land ownership had increased odds of dietary diversity score (DDS) among children 1.11 times [48]. 
Women empowerment's indicator from the agency dimension, which is related to the good practice of IYCF by mothers, is women's involvement in the household decisionmaking process. Two studies mentioned that children whose mothers involved in the household's decision-making process were more likely to meet recommended meal frequency (1.5 times) and dietary diversity (1.27 times), as compared to the children whose mothers did not involve [42], [48]. Moreover, women's autonomy over resources also significantly correlated with a good score of IYCF practice. Studies from Uganda revealed that women with a high level of freedom in the movement were 1.69 times more likely to fulfil children's dietary diversity, as recommended by WHO [43]. The odds of children's DDS and acceptable diet score increase as women have a higher ability to control their lives and financial resources [25], [49].

This review mentioned three indicators of women empowerment from the achievement dimension, which facilitates the IYCF practice, including women's ability to stand against domestic violence, high socioeconomic status, and employment. One study from Ghana stated that the score of women's opinion about wife-beating legitimacy is positively related to children's dietary diversity score [41]. Several studies mentioned that women living in a high socioeconomic status household or higher monthly income rather than average were positively associated with the child's dietary diversity and acceptable diet [30], [31], [37], [38], [48]. Children from mothers living in the highest wealth index household were 5.11 times more likely to consume iron-rich food [38]. Also, one study revealed that a mother's status as a worker also facilitates appropriate IYCF practice. A study conducted in Pakistan found that an employed mother was better at achieving the recommended score of the child's acceptable diet than an unemployed mother [31].

\subsubsection{Women's empowerment as barriers to IYCF practices}

This review also identified studies highlighting women's empowerment indicator which inhibit IYCF practices. Resources, includes low or uneducated [29], [33], [35], [36], [40], [45]-[47], and forced to be the head of household [34]. Agency, includes not involved in household decision making process [35], [47]. Achievement, includes low socioeconomic status [27], [29], [32], [33], [35], [36], [39], [40], [46], [47], and workload [28], [29], [43], [46], [47].

This review identified nine studies on women empowerment's indicator from the resource dimension as a barrier to IYCF practice. Most of the studies mentioned uneducated or low-educated women as a significant predictor for child's poor dietary diversity, unmet minimum meal frequency, and inadequate acceptable diet based on WHO recommendation [29], [33], [35], [36], [40], [45]-[47]. One study from Tanzania found that female-headed households tend to give their children a less diverse diet compared to those in male-headed households [34].
Five studies reported women's empowerment indicators from the agency dimension, which can hinder the IYCF practice by mother. Firstly, women are not involved in the household's decision-making process. Studies conducted in India and Sri Lanka found inadequate dietary diversity and meal frequency happens if the mother was not involved in the household decision-making process [35], [47]. Surprisingly, women's physical and financial autonomy was negatively associated with the three feeding indicators based on WHO guidelines for IYCF. Women with physical autonomy have the freedom to move or travel somewhere, so they cannot keep an eye on the diet of their child who left at home [25], [43]. At the same time, women with the financial economy have the freedom to allocate money to buy food. On the opposite, they tend to buy less diverse food [39].

This review mentioned two indicators of women empowerment from the achievement dimension, which inhibit the IYCF practice, including low socioeconomic status and working women's workload. Several studies conducted in LMIC reported low social-economic status was negatively correlated with the achievement of children's minimum dietary diversity, meal frequency, and acceptable diet. Women from low socioeconomic [36], [39], low monthly income [32], [40], and low wealth categories household [29], [33], [35], [46], [47] were two times less likely to perform IYCF according to WHO recommendation. Lack of purchasing power also inhibits a mother's ability to diversify children's diet [27]. Furthermore, the workload of working women also becomes a barrier to perform a good IYCF practice. Mother who was formally employed or working away was 0.34 to 1.91 times less likely to give their children a proper diverse diet, meal frequency, and acceptable diet [28], [29], [43], [46], [47].

\section{DISCUSSION}

This review found women's empowerment indicators, which act as facilitators and barriers of IYCF practices. Women empowerment defines women's ability to claim the resources available in their household, have control, participate in decision making, and act according to their wishes to change their lives to be better. The indicator of women empowerment is divided into three dimensions: resources, agency, and achievement [20], [23].

The first dimension of women's empowerment is resources. Resources include material/things in the more conventional economic sense and human and an institutional environment that enables women to make decisions, such as education, social capital, and asset ownership [20]. Across 24 studies, well-educated women were highlighted as the essentials indicator that empowers women to perform adequate IYCF practice. There were seven studies found that women with secondary or higher-level education had greater odds of feeding their children based on the WHO guidelines (meet minimum dietary diversity and minimum meal frequency). It is possible, as education plays a vital role in raising women's ability to make decisions [50]. Education 
shapes women to be more confident and refuse disadvantaged traditional beliefs and practices regarding IYCF [51]. It also increases women's awareness to promote appropriate IYCF practice [52]. Women should be supported to finish the secondary level education, at least. To provide them with the ability to decide properly regarding IYCF practices.

Several studies mentioned a sense of leadership and land ownership as an indicator of women's empowerment from the resource dimension, which is significantly related to IYCF practice. Maternal leadership referred to the assertiveness and actions which indicate women's sense of self-security, such as a sense of self as a respected member in the household and self-confidence [39]. Women with high self-confidence are better at determining decisions that enhance health and positively affect their child's nutritional status [53]. On the opposite, women with low self-confidence may have difficulty perceiving the need to perform health care, much less gathering their confidence to act on that need [39]. Moreover, one study in Ethiopia reported that land ownership is also important to increase of high dietary diversity [48]. When women have authority over the resources, their sense of self and self-confidence will also increase. For this reason, it is necessary to strengthen the role of women and their authority within the households, so they will be more confident in performing appropriate IYCF practice for their children.

The second dimension of women's empowerment is an agency. Agency means the ability to think critically and to make decisions independently [20]. This review reported women's involvement in the household decision-making process and women's autonomy over resources to indicate women's empowerment from the agency dimension associated with IYCF practice. In most families, a mother has an essential role in child feeding [54]. So, women's participation in their household issues is important, as they have easy and free access to their household resources, they can feed their children more frequently, according to WHO recommendation. Women's autonomy referred to women's freedom to make choices and control over their life (movement, finance, and others) [25], [49]. As women have the autonomy to choose, they can choose the right or wrong thing regarding IYCF practices [25]. Women who had financial freedom can allocate money and purchase food for their children's benefit [39]. Women who had mobility freedom can travel to the market to buy healthy food for their children [43], [49]. So, mothers should use their freedom as much as possible to meet the nutritional needs of children.

The third dimension of women's empowerment is an achievement. Achievement is a manifestation of using the agency, for example, employment, not tolerating domestic violence, and providing time for childcare [23]. The high socioeconomic status of the household is also mentioned in five studies as an indicator of women's empowerment, which facilitates IYCF practices. At the same time, ten studies found that low socioeconomic status can inhibit IYCF practices. Women from the household with high socioeconomic status were more likely to improve dietary diversity, higher frequency of food intake, and meet minimum acceptable diet in feeding their children. Higher socioeconomic status means higher monthly income and a higher ability to choose healthy food [55]. Living in a high socioeconomic status household is an important indicator of women's empowerment from the achievement dimension, empowering women to provide appropriate IYCF practices for their children.

This review also found women's workload as an indicator of women empowerment, which inhibits IYCF practices. Five studies mentioned that working mother was more likely unmet minimum meal frequency and acceptable diet. Mothers also reported that they were feeding their children with cheap processed food that was not adequate in nutrition [28].

The limitation of this systematic review includes the exclusion of paper not published in English due to author's limitations which would have added the diversity of findings, did not explore grey literature which may relate to the study topics, and did not involve a librarian to help in searching potential articles for this review. These limitations mean this review is possibly not comprehensive. However, this review can be used as an evidence to guide research in the future, to improve health promotion strategies, and to enhance public policy regarding women empowerment and IYCF practice. The main strength of this review is that we covered studies from the different region of LMIC. We also searched studies from five top-tier databases, makes it possible to get high quality research.

\section{CONCLUSIONS}

Well-educated women and high socioeconomic status of the household is an important indicator of women's empowerment for appropriate IYCF practice. Women's involvement in the household decision-making process was also needed to ensure the household's resources to support optimal IYCF practices. Although women's employment can increase income, which facilitates the IYCF practices, their workload should be limited, so they still have time to provide optimal IYCF practices to their children. This review also identified women's autonomy over resources to indicate women's empowerment, facilitating, and inhibiting IYCF practices. Future research and empowerment intervention to improve IYCF should address these findings.

\section{AUTHORS' CONTRIBUTIONS}

NN contributed to the concept, study design, thematic analysis, and finalized the essential intellectual contents. EMMH and YSA performed article search, data extraction, thematic analysis, and data interpretation. All authors equally write the draft, read, and approved the final manuscript.

\section{ACKNOWLEDGMENTS}

The authors would like to thank Universitas Airlangga, which provides access to the databases, and the Ministry of Research 
and Technology, The Republic of Indonesia, which gives financial support for this review through "Penelitian Disertasi Doktor" scheme (Grant number 349/DRPM/thp-I/2020).

\section{REFERENCES}

[1] WHO, "Malnutrition," 2020. .

[2] UNICEF, WHO, and World Bank, "Levels and trends in child malnutrition: Key findings of the 2020 Edition of the Joint Child Malnutrition Estimates.," 2020.

[3] Development Initiatives, "Global Nutrition Report 2020," Bristol, UK, 2020.

[4] UNICEF, “Global Nutrition Report 2020: Action on equity to end malnutrition," 2020 . .

[5] R. E. Black et al., "Maternal and child undernutrition and overweight in low-income and middle-income countries," Lancet, vol. 382, no. 9890, pp. 427-451, 2013.

[6] P. S. Suchdev, "What Pediatricians Can Do to Address Malnutrition Globally and at Home," Pediatrics, vol. 139, no. 2, p. e20161666, 2017.

[7] T. Gödecke, A. J. Stein, and M. Qaim, "The global burden of chronic and hidden hunger: Trends and determinants," Glob. Food Sec., vol. 17, no. March, pp. 21-29, 2018.

[8] N. Nisbett, S. Gillespie, L. Haddad, and J. Harris, "Why worry about the politics of childhood undernutrition?," World Dev., vol. 64, pp. 420-433, 2014.

[9] WHO, "What is malnutrition?," WHO Publication, 2016. .

[10] I. M. Mitchodigni et al., "Complementary feeding practices: determinants of dietary diversity and meal frequency among children aged 6-23 months in Southern Benin," Food Secur., vol. 9, no. 5, pp. 1117-1130, 2017.

[11] P. Menon, "The crisis of poor complementary feeding in South Asia: where next?," Matern. Child Nutr., vol. 8, no. Suppl 1, p. 1, 2012.

[12] V. J. B. Martins, T. M. M. T. Florê, C. D. L. Santos, M. D. F. A. Vieira, and A. L. Sawaya, "Long-Lasting Effects of Undernutrition," Int. J. Environ. Res. Public Health, vol. 8, pp. 1817-1846, 2011.

[13] V. Khanal, K. Sauer, and Y. Zhao, "Determinants of complementary feeding practices among Nepalese children aged 6-23 months: Findings from demographic and health survey 2011," BMC Pediatr., vol. 13 , no. $1,2013$.

[14] WHO, Indicators for assessing infant and young child feeding practices: part 2 measurement. Geneva: WHO, 2010.

[15] F. A. Olatona, J. O. Adenihun, S. A. Aderibigbe, and
O. F. Adeniyi, "Complementary Feeding Knowledge, Practices, and Dietary Diversity among Mothers of Under-Five Children in an Urban Community in Lagos State, Nigeria," Int. J. MCH AIDS, vol. 6, no. 1, pp. 46-59, 2017.

[16] S. Elliott and S. Bowen, "Defending motherhood: Morality, responsibility, and double binds in feeding children," J. Marriage Fam., vol. 80, no. 2, pp. 499520,2018

[17] I. Moreira et al., "Social and health behavioural determinants of maternal child-feeding patterns in preschool-aged children," Matern. Child Nutr., vol. 12, no. 2, pp. 314-325, 2016.

[18] R. Jones, R. Haardorfer, U. Ramakrishnan, K. M. Yount, S. Miedema, and A. Webb Girard, "Women's empowerment and child nutrition: The role of intrinsic agency," SSM-Population Heal., vol. 9, no. September 2018, pp. 1-11, 2020.

[19] T. Tandon, "Women Empowerment: Perspectives and Views," Int. J. Indian Psychol., vol. 3, no. 3, p. 6, Jan. 2016.

[20] N. Kabeer, "Resources, Agency, Achievements : Re ections on the Measurement of Women' $\mathrm{s}$ Empowerment," Dev. Change, vol. 30, no. May, pp. 435-464, 1999.

[21] K. Cunningham et al., "Women's empowerment in agriculture and child nutritional status in rural Nepal," Public Health Nutr., vol. 18, no. 17, pp. 3134-3145, 2015.

[22] K. S. Imai, S. K. Annim, V. S. Kulkarni, and R. Gaiha, "Women's Empowerment and Prevalence of Stunted and Underweight Children in Rural India," WORLD Dev., vol. 62, no. July 2012, pp. 88-105, 2014.

[23] M. V Santoso, R. B. Kerr, J. Hoddinott, P. Garigipati, S. Olmos, and S. L. Young, "Role of Women"'s Empowerment in Child Nutrition Outcomes: A Systematic Review," Adv. Nutr., vol. 10, pp. 1138 $1151,2019$.

[24] A. Siddhanta and A. Chattopadhyay, "Role of Women's Empowerment in Determining Child Stunting in Eastern India and Bangladesh," vol. 3, no. 1, pp. 38-51, 2017.

[25] M. Na, L. Jennings, S. A. Talegawkar, and S. Ahmed, "Association between women's empowerment and infant and child feeding practices in sub-Saharan Africa: an analysis of Demographic and Health Surveys.," Public Health Nutr., vol. 18, no. 17, pp. 3155-3165, Dec. 2015.

[26] D. Moher, A. Liberati, J. Tetzlaff, and D. G. Altman, "Preferred reporting items for systematic reviews and meta-analyses: the PRISMA statement," Int J Surg, vol. 8, no. 5, pp. 336-341, 2010.

[27] J. Burns, J. A. Emerson, K. Amundson, S. Doocy, L. 
E. Caulfield, and R. D. W. Klemm, "A qualitative analysis of barriers and facilitators to optimal breastfeeding and complementary feeding practices in South Kivu, Democratic Republic of Congo," Food Nutr. Bull., vol. 37, no. 2, pp. 119-131, 2016.

[28] A. Kabir and M. R. L. Maitrot, "Factors influencing feeding practices of extreme poor infants and young children in families of working mothers in Dhaka slums: A qualitative study," PLoS One, vol. 12, no. 2, p. e0172119, 2017.

[29] U. Senarath et al., "Comparisons of complementary feeding indicators and associated factors in children aged 6-23 months across five South Asian countries," Matern. Child Nutr., vol. 8, no. SUPPL. 1, pp. 89106, 2012.

[30] K. Karwa, M. L. Godhia, and A. R. Jadhav, "Infant and Young Child-Feeding Practices, Indicators and Index, and Role of Socio-economic Status," Int. J. Adv. Nutr. Heal. Sci., vol. 4, no. 1, pp. 212-218, 2016.

[31] G. N. Khan et al., "Determinants of infant and young child feeding practices by mothers in two rural districts of Sindh, Pakistan: A cross-sectional survey," Int. Breastfeed. J., vol. 12, no. 1, pp. 1-8, 2017.

[32] G. Kumera, E. Tsedal, and M. Ayana, "Dietary diversity and associated factors among children of Orthodox Christian mothers/caregivers during the fasting season in Dejen District, North West Ethiopia," Nutr. Metab., vol. 15, no. 1, 2018.

[33] C. S. Ng, M. J. Dibley, and K. E. Agho, "Complementary feeding indicators and determinants of poor feeding practices in Indonesia: A secondary analysis of 2007 Demographic and Health Survey data," Public Health Nutr., vol. 15, no. 5, pp. 827839, 2012.

[34] J. Ochieng, V. Afari-Sefa, P. J. Lukumay, and T. Dubois, "Determinants of dietary diversity and the potential role of men in improving household nutrition in Tanzania," PLoS One, vol. 12, no. 12, p. e0189022, 2017.

[35] A. Patel, Y. Pusdekar, N. Badhoniya, J. Borkar, K. E. Agho, and M. J. Dibley, "Determinants of inappropriate complementary feeding practices in young children in India: Secondary analysis of National Family Health Survey 2005-2006," Matern. Child Nutr., vol. 8, no. SUPPL. 1, pp. 28-44, 2012.

[36] N. H. Rakotonirainy et al., "Dietary diversity of 6- to 59-month-old children in rural areas of Moramanga and Morondava districts, Madagascar," PLoS One, vol. 13, no. 7, pp. 1-14, 2018.

[37] D. Solomon, Z. Aderaw, and T. K. Tegegne, "Minimum dietary diversity and associated factors among children aged 6-23 months in Addis Ababa, Ethiopia," Int. J. Equity Health, vol. 16, no. 1, pp. 19, 2017.
[38] C. N. Walters, H. Rakotomanana, J. J. Komakech, and B. J. Stoecker, "Maternal determinants of optimal breastfeeding and complementary feeding and their association with child undernutrition in Malawi ( 2015 - 2016 )," pp. 1-12, 2019.

[39] H. Alaofé et al., “Association Between Women's Empowerment and Maternal and Child Nutrition in Kalalé District of Northern Benin.," Food Nutr. Bull., vol. 38, no. 3, pp. 302-318, Sep. 2017.

[40] A. Wang et al., "The dietary diversity and stunting prevalence in minority children under 3 years old: A cross-sectional study in forty-two counties of Western China," Br. J. Nutr., vol. 118, no. 10, pp. 840-848, 2017

[41] D. A. Amugsi, M. B. Mittelmark, and A. Oduro, "Association between maternal and child dietary diversity: An analysis of the Ghana Demographic and Health Survey," PLoS One, vol. 10, no. 8, pp. 1-12, 2015.

[42] M. Beyene, A. G. Worku, and M. M. Wassie, "Dietary diversity, meal frequency and associated factors among infant and young children in Northwest Ethiopia: A cross-sectional study," BMC Public Health, vol. 15, no. 1, 2015.

[43] S. B. Ickes, T. E. Hurst, and V. L. Flax, "Maternal Literacy, Facility Birth, and Education Are Positively Associated with Better Infant and Young Child Feeding Practices and Nutritional Status among Ugandan Children," J. Nutr., vol. 145, no. 11, pp. 2578-2586, 2015.

[44] S. B. Ickes, M. Wu, M. P. Mandel, and A. C. Roberts, "Associations between social support, psychological well-being, decision making, empowerment, infant and young child feeding, and nutritional status in Ugandan children ages 0 to 24 months," Matern. Child Nutr., vol. 14, no. 1, pp. 1-11, 2018.

[45] B. E. Isingoma, M. Samuel, K. Edward, and G. W. Maina, "Socioeconomic and Demographic Factors Influencing Feeding Practices, Morbidity Status, and Dietary Intakes of Children Aged 7-24 Months in Rural Uganda," Ecol. Food Nutr., vol. 56, no. 1, pp. 1-16, 2017.

[46] N. Joshi, K. E. Agho, M. J. Dibley, U. Senarath, and K. Tiwari, "Determinants of inappropriate complementary feeding practices in young children in Nepal: Secondary data analysis of Demographic and Health Survey 2006," Matern. Child Nutr., vol. 8, no. SUPPL. 1, pp. 45-59, 2012.

[47] U. Senarath, S. S. P. Godakandage, H. Jayawickrama, I. Siriwardena, and M. J. Dibley, "Determinants of inappropriate complementary feeding practices in young children in Sri Lanka: Secondary data analysis of Demographic and Health Survey 2006-2007," Matern. Child Nutr., vol. 8, no. SUPPL. 1, pp. 60-77, 2012. 
[48] D. Kuche et al., "Factors associated with dietary diversity and length-for-age z-score in rural Ethiopian children aged 6-23 months: A novel approach to the analysis of baseline data from the Sustainable Undernutrition Reduction in Ethiopia evaluation," Matern. Child Nutr., vol. 16, no. 1, pp. 1-11, 2020.

[49] S. B. Ickes, G. A. Heymsfield, T. W. Wright, and C. Baguma, "'Generally the young mom suffers much:' Socio-cultural influences of maternal capabilities and nutrition care in Uganda," Matern. Child Nutr., vol. 13, no. 3, pp. 1-13, 2017.

[50] M. K. Khatwani, I. A. Abbasi, and F. Y. Panhwar, "Professional Women's Perception about Role of Higher Education in Decision-Making Process," Grassroots, vol. 52, no. 1, pp. 144-156, 2018.

[51] G. T. Kiross, C. Chojenta, D. Barker, T. Y. Tiruye, and D. Loxton, "The effect of maternal education on infant mortality in Ethiopia: A systematic review and meta-analysis," PLoS One, vol. 14, no. 7, p. e0220076, 2019.

[52] A. H. Dagne, K. T. Anteneh, M. B. Badi, H. H. Adhanu, M. A. Ahunie, and G. L. Aynalem, "Appropriate complementary feeding practice and associated factors among mothers having children aged 6-24 months in Debre Tabor Hospital, North West Ethiopia, 2016," BMC Res. Notes, vol. 12, no. 1, pp. 1-6, 2019.

[53] K. L. Ross, Y. A. Zereyesus, A. Shanoyan, and V. Amanor-Boadu, "The health effects of women empowerment: Recent evidence from northern Ghana," Int. Food Agribus. Manag. Rev., vol. 18, no. 1, pp. 127-144, 2015.

[54] I. U. Eneli, T. L. Tylka, R. P. Watowicz, and J. C. Lumeng, "Maternal and child roles in the feeding relationship: what are mothers doing?," Clin. Pediatr. (Phila)., vol. 54, no. 2, pp. 179-182, Feb. 2015.

[55] S. A. French, C. C. Tangney, M. M. Crane, Y. Wang, and B. M. Appelhans, "Nutrition quality of food purchases varies by household income: the SHoPPER study," BMC Public Health, vol. 19, no. 1, p. 231, 2019. 